\title{
Establishing Agrobacterium mediated transient gene expression assay in Nicotiana tabacum
}

\author{
Muhammad Rafique Rind ${ }^{1 *}$, Aneela Yasmin ${ }^{1,2}$, Muharam Ali $^{1}$ and \\ Saboohi Raza ${ }^{3}$ \\ 1. Department of Biotechnology, Sindh Agriculture University, Tandojam-Pakistan \\ 2. USPCASW, Mehran University of Engineering and Technology, Jamshoro-Pakistan \\ 3. Department of Agriculture and Agribusiness Management, University of Karachi-Pakistan \\ *Corresponding author's email: rindrafique@ gmail.com
}

Citation

Muhammad Rafique Rind, AneelaYasmin, Muharam Ali and Saboohi Raza. Establishing Agrobacterium based transient gene expression assay in Nicotiana tabacum. Pure and Applied Biology. Vol. 7, Issue 4, pp 1286-1293. http://dx.doi.org/10.19045/bspab.2018.700149

\begin{tabular}{llll}
\hline \hline Received: 25/05/2018 & Revised: 03/09/2018 & Accepted: 03/09/2018 & Online First: 06/09/2018 \\
\hline
\end{tabular}

\section{Abstract}

Preliminary functional characterization of a gene within a short time period is only possible through transient gene expression assays. Transient gene expression assays are simple, cost effective and easy to perform as compared to generation of stable transformants. Here we report an Agro-infiltration based transient gene expression assay, optimized in Nicotiana tabacum using $\beta$-glucuronidase (GUS) intron gene as an indicator to evaluate various factors effecting its expression. The factors evaluated include bacterial concentration and its growth mode, infiltration medium, concentration of acetosyringone and surfactant, co-cultivation time of Agrobacterium and host cells and temperature during co-cultivation. When Agrobacteria were grown in/ on YEP liquid and solid media, both mode of growth did not affect the transformation efficiency of bacteria and it was recorded $100 \%$ in both cases. However, the transient gene expression efficiency found optimal when fully expended true leaves of tobacco infiltrated with Agrobacterium strain EHA101 carrying GUS intron marker gene in $0.3 \%$ glucose medium with an optical density $\left(\mathrm{OD}_{600}\right)$ of 0.3 without acetosyringone and surfactant. After infiltration samples were stored at $22^{\circ} \mathrm{C}$ for two days and evaluations were carried out after histochemical staining of agro-infiltrated discs by visual inspection. This optimized protocol gave the highest level of GUS expression- 3 in 66.7\% of samples, followed by 33.0\% (GUS expression level -2) and 0\% (GUS expression level-1) of samples. The simplicity of this method suggests its utility for the production of pharmaceutical proteins in native plants of Pakistan and can help to promote biotechnology awareness at schools and colleges.

Keywords: Agrobacterium mediated transient expression; $\beta$-glucuronidase; Nicotiana tabacum Introduction

Genetic transformation is a powerful tool to introduce new traits in elite germplasm and functionally characterize new genes. During this process usually exotic altered DNA is introduced into a eukaryotic or prokaryotic cell followed by assessing the function and effect of its products on cellular level or on organism level [1]. Nevertheless, isolating a gene then transporting it into a cell without 
destroying the cell is also a challenge for molecular scientists. The delivery of gene of interest (GOI) in a cell can be mediated by biolistic gun, microinjection, agrobacterium, electroporation, silicon carbide fiber, liposome, calcium phosphate coprecipitation, polybrene spermidine treatment etc. $[2,3]$. Depending on the objective genetic transformation could be stable or transient. Stable transgenic are generated by introducing GOI into the cells that need to be integrated into the genome of the host. In reality only a small portion of targeted cells get GOI integrated in their genomes. The resultant stable transformants should be having GOI as a part of its genome that can replicate and transfer the expressing GOI to offspring [4]. Although stable transgenic express the gene of interest stably, it requires long term screening, laborious analysis and vigilant handling of potential transgenic for months. The expression variability is common in stable transgenic lines due to the copy number, silencing or insertion location of GOI in the host genome [5]. In addition to GOI a reporter gene is fused with it to know if the cells/tissues are transformed or not [6]. Thus, reporter genes report the presence of GOI in the transformants.

In contrast, ectopic transient gene expression can be used to study the function of GOI in short time, within days [7]. This is due to the expression of various copies of nonintegrated GOI present in the host nucleus for first several days after the delivery of GOI into the host cells [8]. As these nonintegrated copies of GOI cannot replicate, these will be lost with time not due to the degradation of non-integrated T-DNA copies but also as silencing process. It is demonstrated by infiltration of T-DNA carrying a GOI and a viral silencing suppressor gene that extended transient expression for as many as seven days post infiltration [9, 10]. According to [5], the transient expression could be thousand times more powerful than in stable transgenics. There are different methodologies available for ectopic gene expression studies in plants namely electroporation or, polyethylene glycol (PEG) based transformation of protoplasts, biolistics and agro-infiltration [2, 3]. Among these methods electroporation or, polyethylene glycol (PEG) based transformation of protoplasts are optimized for few plant species [11]. In this regard, initial preparations like generating protoplasts is also time consuming whereas biolistic machines and consumables are expensive. However, agro-infiltration is a less expensive, less laborious and an ideal method for many plant species [4]. Transient gene expression has been utilized for a wide range of applications like demonstrating promoter activity, function of newly identified genes and proteins, evaluating virulence capacity of wild strains of agrobacterium, protein interactions in vivo and mass production of pharmaceuticals [12$15]$.

Agroinfiltration is a simple and popular tool to effectively study the transgenes' expression into a heterologous system like in tobacco. However, despite its popularity, there is little work done on the improvement of its efficiency to further increase the level of gene expression or protein yields. Keeping in view the usability and simplicity of transient gene expression we report here an efficient, reproducible, and relatively simple methodology for transient gene expression of GUS gene in a locally cultivated plant species of tobacco, namely Nicotiana tabacum. This optimized assay can be utilized for ectopic expression studies of newly identified genes. Further implications include genetic complementation, expression of resistance genes, RNAi experimentations, Protein trafficking and production. To our knowledge this is the first report on utilization of Nicotiana tabacum based 
transient gene expression assay in Sindh, Pakistan.

\section{Materials and methods}

Tobacco, (Nicotiana tabacum) plants were grown for 6-weeks and counting from the upper side of plant 1-4 fully expended true leaves were used in present study. Agrobacterium strain used in present study was EHA 101 (pIG121-Hm) carrying $\beta$ glucuronidase (GUS) gene.

Bacteria were grown and used for agroinfiltration as described by $[7,8]$, with minor modifications. Sterile YEP (10 $\mathrm{g} \mathrm{L}^{-1}$ yeast extract, $5 \mathrm{~g} \mathrm{~L}^{-1}$ sodium chloride, $10 \mathrm{~g} \mathrm{~L}^{-1}$ peptone, $\mathrm{pH} 7.5$, agar $1.5 \%$ ) solid and liquid media were used to grow bacteria. Liquid YEP media was inoculated by a single colony of bacteria and were allowed to grow until bacterial density reached the OD $(600 \mathrm{~nm})$ $1.0-1.5$ at $180 \mathrm{rpm}$. Then the bacteria were harvested by centrifugation at $4500 \mathrm{rpm}$ for $10 \mathrm{~min}$, washed in distilled sterile water at RT and bacterial suspensions were prepared for infiltration, accordingly. In addition bacteria were grown on YEP agar plates until complete bacteria lawn is formed on plate then these bacteria were removed from plates, washed in distilled sterile water at RT, centrifuged at $4500 \mathrm{rpm}$ for $10 \mathrm{~min}$ and bacterial suspensions were prepared for infiltration, accordingly. All leaves were infiltrated by a $1 \mathrm{ml}$ needle without syringe and samples were kept in dark at $22^{\circ} \mathrm{C}$. Histochemical staining was performed as described by [16]. Leaf discs were merged in staining solution and samples were incubated at $37^{\circ} \mathrm{C}$ in dark, overnight. Next day discs were washed in $75 \%$ ethanol to remove the green chlorophyll.

The parameters studied to optimize GUS gene expression in tobacco were bacterial growth mode (solid and liquid), bacterial density $\left(\mathrm{OD}_{600} 0.0,0.3,0.5,1.0\right)$, infiltration medium $(0,0.3 \%$, or $0.5 \%$ glucose medium $)$, the effect of acetosyringone $(0,100 \mu \mathrm{M})$, surfactant (Tween $20-0.0,0.01 \%$ ) and co- cultivation time of Agrobacterium and host cells (0, 2, 5- days). Each experiment was conducted as randomized complete block design with three replications each containing at least 10 leaf discs. The effect of different parameters was recorded in the form of percent transformation efficiency (GUS expression showing discs/total discs inoculated * 100) and level of gene expression. Level of gene expression was rated by visual inspection of inoculated discs after staining and rating for observed blue colour (GUS expression) on a scale mentioned in (Figure 1).

\section{Results and discussion}

An Agro-infiltration based transient gene expression assay was optimized in model plant Nicotiana tabacum. Agrobacterium strain EHA101 was harboring $\beta$ glucuronidase (GUS) intron gene that was used as an indicator to evaluate various factors effecting its expression. Among evaluated factors first bacterial concentration, infiltration medium, and cocultivation time was optimized. Then holding these factors constant effects of bacterial growth mode, surfactant and acetosyringone on GUS expression were evaluated.

Density of bacterial suspension is an important factor to show gene expression [7]. All the tested bacterial concentrations ( $\mathrm{OD}_{600}$ $0.3,0.5,1.0)$ displayed $100 \%$ transformation efficiency however the level of expression was variable (Table 1, Figure $3 \mathrm{~A}$ ). It is interesting to notify that the bacterial densities $\mathrm{OD}_{600} 0.3$ showed the highest level of GUS expression that is 3 in $66.7 \%$ (Fig.3A) of samples followed by $\mathrm{OD}_{600} 0.5$ (23.3\%; Figure 3A) and $\mathrm{OD}_{600} 1.0$ (10.0\%; Figure $3 \mathrm{~A}$ ) thus we finalize $\mathrm{OD}_{600} 0.3$ as optimal concentration of bacteria for GUS expression in tobacco. It is due to the fact that Agrobacterium is a plant pathogen that transfers its T-DNA to the host genome and induces plant defenses in Arabidopsis [17, 18], grapevine [6], suppresses plant defenses 
in tobacco cell cultures [19]. In present study as the bacterial concentration was increased to $\mathrm{OD}_{600} 1.0$ the texture of leaf was effected negatively and wilting was observed furthermore the expression level was also affected negatively (Figure 3A). The observed levels of GUS expression i.e. GUS 1,2 and 3 at OD $_{600} 1.0$ were $40 \%, 50 \%$ and $10 \%$, respectively. In addition to bacterial strain, bacterial concentration or density can also affect the infection process and thus the transformation efficiencies. Low amount of bacteria in a suspension will result in low bacteria and host cell ratio and low transformation frequencies whereas high bacterial concentration will damage the tissues and revealing low transformation frequencies [20].

Previously it was shown that different infiltration media [21], effect the expression positively. In present study we also observed the positive effect of glucose as infiltration media (Figure 3B). Glucose at the concentration of $0.3 \%$ improved the expression level of all tobacco discs from 2 to 3 and none of the evaluated discs revealed $1^{\text {st }}$ level of GUS expression. Thus infiltration medium was found critical for improving gene expression in present study. Although the transformation efficiency was $100 \%$ when bacterial suspension was prepared in distilled sterile water also, the highest GUS expression level was recorded as 01 (Figure $3 \mathrm{~A} ; 50 \%$ ) that was increased to 3 (Figure 3A; $66 \%$ ) when $0.3 \%$ glucose solution was used as infiltration medium. As the concentration of glucose in infiltration medium was increased to $0.5 \%$ the highest expression level 3 is also decreased to 50\%. However, there are reports demonstrating no effect of infiltration media on the gene expression but these finding are in different plants like rose [7], N. benthamiana, Arabidopsis, tomato and lettuce [4]. Moreover addition of acetosyringone [8].or surfactants [22] is also reported to improve the transient gene expression significantly. Acetosyringone is added to infiltration media to induce virulence genes of Agrobacterium [23] whereas; surfactants facilitate the infiltration of bacterial suspension into the host tissues [22]. In the present study neither acetosyringone $(100 \mu \mathrm{M})$ nor surfactant (Tween-20; concentration $0.01 \%$ ) improved the transient GUS gene expression in $N$. tabacum significantly (Figure 3C, 3D). Same observations recorded in Arabidopsis and lettuce [4]. Agroinfiltration is a tool to remarkably facilitate the studies of functional characterization of newly identified genes meanwhile Agrobacterium is actually a plant pathogen and elicits immune responses in host also [24].

When Agrobacteria were grown in/ on YEP liquid and solid media, both mode of growth did not affect the transformation efficiency of bacterial that was $100 \%$ in both cases and the highest GUS expression level was found as 3 on visual scale (Figure 3E; 66.7\%). This finding will provide scientists, a choice as that they can use any medium for the growth of bacteria for agro-infiltration studies [25]. Nonetheless, the co-cultivation of host and Agrobacterium was evaluated on day 0, 2 and 5 . The day 0 was the same day of infiltration. Within first hour of infiltration of Agrobacterium suspension in the host did not revealed any gene expression however, gene expression level was found non-significantly different when staining was carried out after day 2 or day 5 (Figure 3F). 


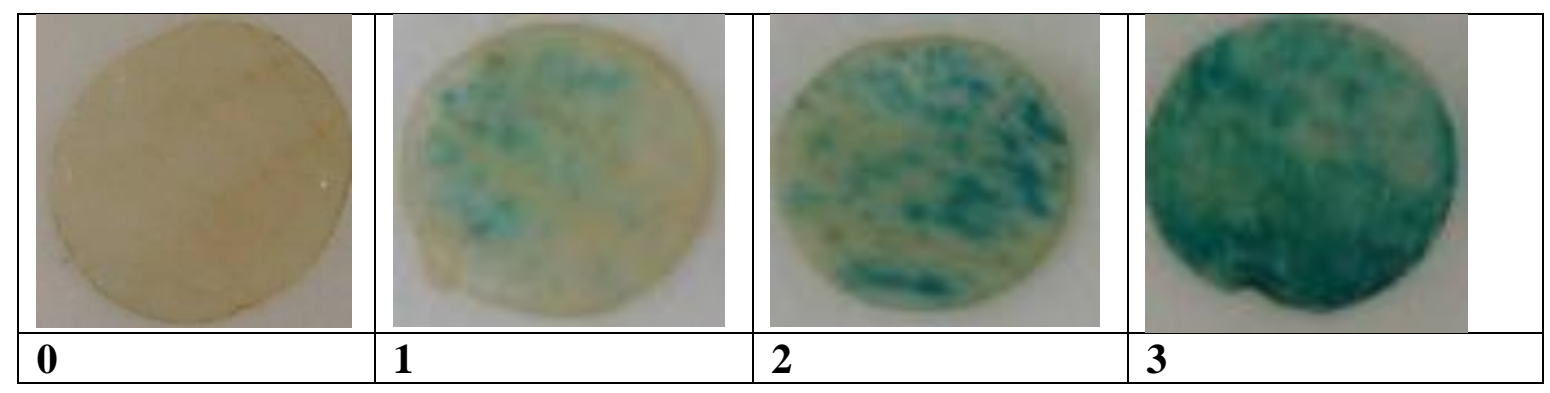

Figure 1. A visual scale to categorize different levels of GUS expression in tobacco

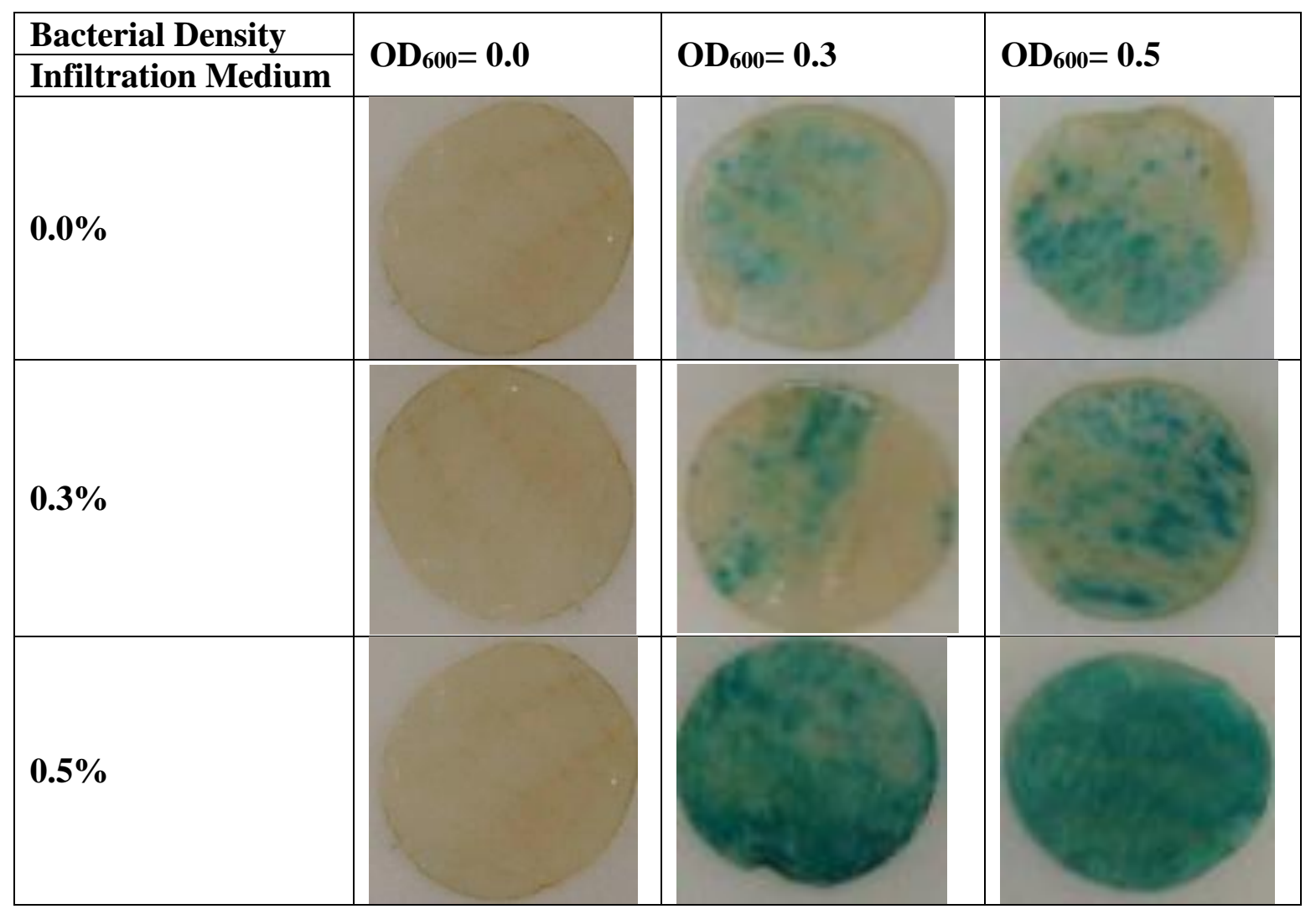

Figure 2. Effect of different parameters on transient GUS expression

Table 1. Percent transformation efficiency of GUS infiltrated tobacco discs

\begin{tabular}{|c|c|}
\hline $100 \%$ transformation efficiency & $0 \%$ transformation efficiency \\
\hline $\begin{array}{c}\text { Bacterial density (O.D } 600-0.3,0.5,1.0) \\
\text { Infiltration medium }(0,0.3,0.5 \%) \\
\text { Co-cultivation time of host and bacteria }(2,5 \text { days) } \\
\text { Surfactant (Tween-20: } 0,0.01 \%) \\
\text { Acetosyringone }(0,100 \mu \mathrm{M}) \\
\text { Bacterial growth mode (solid, liquid) }\end{array}$ & $\begin{array}{c}\text { Bacterial density (O.D } 600-0) \\
\text { Co-cultivation time of host and bacteria } \\
\text { (same days post infiltration) }\end{array}$ \\
\hline
\end{tabular}

Note: Percentage represents three replications each containing at least 10 leaf discs 

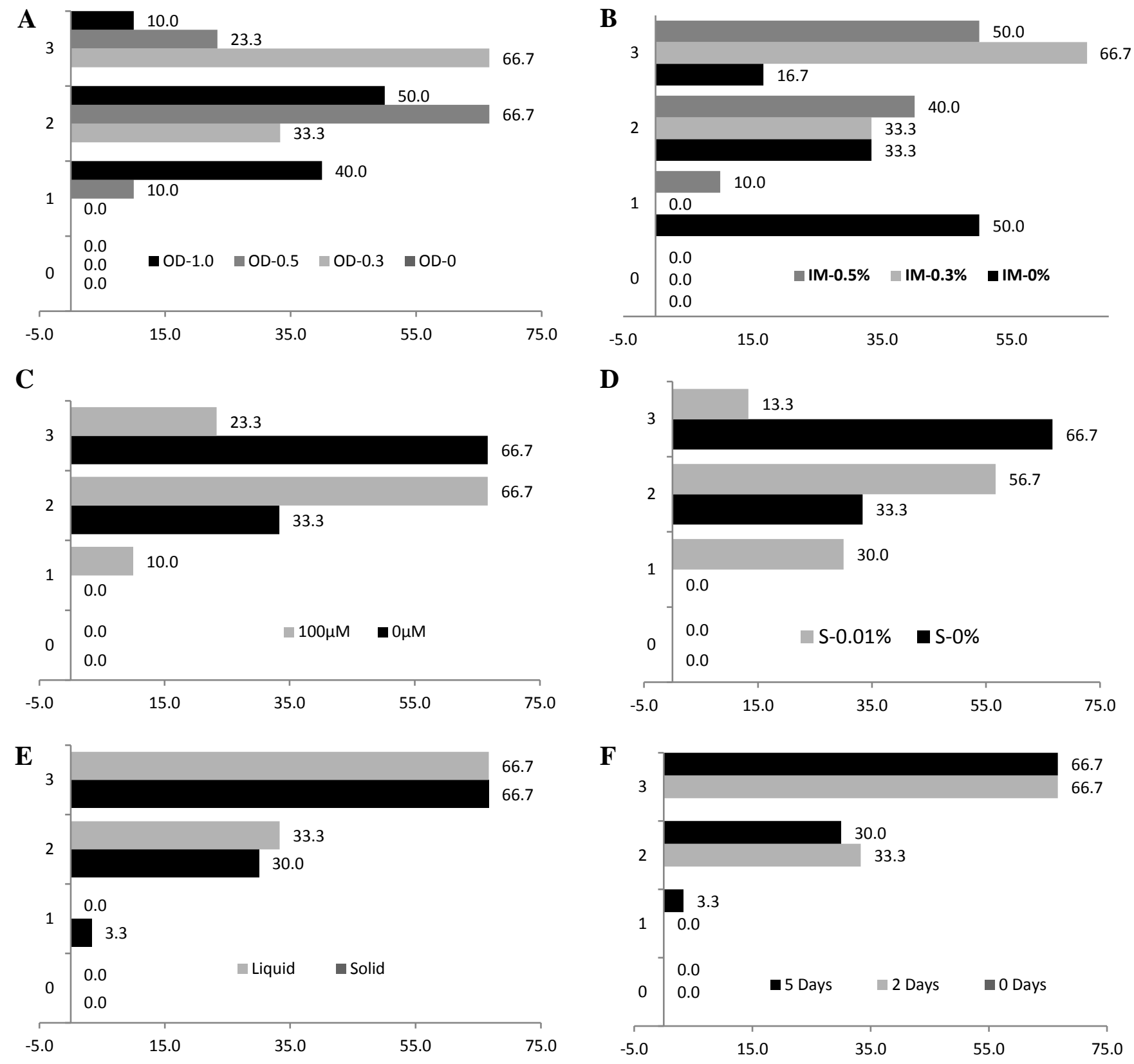

Figure 3. X-Axis and Y-Axis here represent percentage of tobacco discs revealed GUS expression and different levels of GUS expression based on a visual scale provided in Fig. 1, respectively. All graphs reveal the effect of different parameters on the level of GUS expression; A: effect of bacterial concentration; B: Effect of different concentrations of glucose as infiltration medium; C: Effect of acetosyringone; D: Effect of surfactant; E: Effect of bacterial growth mode; E: Effect of bacteria and host co-cultivation period 


\section{Conclusion}

In present study the transient GUS gene expression was optimized. The optimal GUS expression was detected, when bacterial concentration maintained as $0.3 \mathrm{OD}_{600}$ in $0.3 \%$ glucose solution without additives like acetosyringone and surfactant in N. tabacum. The samples were evaluated after 2-days of infiltration that resulted the highest level of GUS expression level - 3 in $66.7 \%$ of samples, followed by $33.0 \%$ (GUS expression level - 2) and 0\% (GUS expression level -1) of samples. This syringe based agro-infiltration method is a simple, less expensive and efficient method for transient expression of genes and its protein that can revolutionize the production of pharmaceutical proteins in a local plant adapted to our environmental conditions. Additionally, the simplicity of this method also suggests its utility to promote biotechnology education at schools and colleges.

\section{Authors' contributions}

Conceived and designed the experiments: A Yasmin; Performed the experiments: MR Rind; Analyzed the data: A Yasmin; Wrote the paper: MR Rind \& A Yasmin.

\section{Acknowledgments}

The authors are thankful to Higher Education Commission of Pakistan (HEC) for the award of indigenous scholarship to M. Rafique for Ph.D.

\section{References}

1. Rao A, Bakhsh QA, Kiani S, Shahzad K, Shahid, AA, Husnain T \& Riazuddin S (2009). The myth of plant transformation. Biotechnol Adv 27: 753763.

2. Songstad DD, Somers DA \& Griesbach RJ (1995). Advances in alternative DNA delivery techniques. Plant Cell Tissue Organ Culture 40: 1-15.

3. Newell CA (2000). Plant transformation technology, developments and applications.
Molecular Biotechnology 16(1): 53-65.

4. Wroblewski T, Tomczak A \& Michelmore R (2005). Optimization of Agrobacterium mediated transient expression assays for lettuce, tomato and Arabidopsis. Plant Biotechnol J 3: 259273.

5. Janssen BJ \& Gardner RC (1990). Localized transient expression of GUS in leaf discs following co-cultivation with Agrobacterium. Plant Mol Biol 14: 6172.

6. Santos R, Poutaraud M, Merdinoglu A \& Mestre D P (2008). Development of a transient expression system in grapevine via Agroinfiltration. Plant Cell Rep 27: 1053-1063.

7. Yasmin A \& Debener $T$ (2010). Transient gene expression in rose petals via Agrobacterium infiltration. Plant Cell Tissue and Organ Culture 102(2): 245-250.

8. Kapila J, Rycke RD, Montagu MV \& Angenon G (1997). An Agrobacteriummediated transient gene expression system for intact leaves. Plant Sci 122: 101-108.

9. Sabalza M, Madeira L, Madeira V, Dolleweerd J, Ma K, Capell T \& Christoup (2012). Functional characterization of the recombinant HIV-neutralizing monoclonal antibody 2F5 produced in maize seeds. Plant Mol Biol 80: 477-488.

10. Cazzonelli CI \& Velten, J (2006). An In vivo, luciferase-based, Agrobacteriuminfiltration assay system and implications for post-transcriptional gene silencing. Planta 224: 582-97.

11. Yoo S D, Cho $\mathrm{Y} H$ \& Sheen $J$ (2007) Arabidopsis mesophyll protoplasts: a versatile cell system for transient gene expression analysis. Nature Protocols 2:1565-1572.

12. Yang $\mathrm{Y}, \mathrm{Li} \mathrm{R} \&$ Qi M (2000). In vivo analysis of plant promoters and 
transcription factors by Agro infiltration of tobacco leaves. Plant J 22: 543-51.

13. Song GQ \& Yamaguchi KI (2003). Efficient agro-infiltration-mediated transient GUS expression system for assaying different promoters in rice. Plant Biol 20: 235-239.

14. Machens F, Becker M, Umrath F \& Hehl $R$ (2014). Identification of a novel type of WRKY transcription factor binding site in elicitor-responsive cis sequences from Arabidopsis thaliana. Plant Mol Biol 84: 371-85.

15. Torregrosa L, Verries $C \&$ Tesniere $C$ (2002). Grapevine (V. vinifera L.) promoter analysis by biolistic-mediated transient transformation of cell suspensions. J of Grapevine Res 41: 2732.

16. Jefferson RA, Kavanagh TA \& Bevan MV (1987). GUS fusions: $\beta$ glucuronidase as a sensitive and versatile gene fusion marker in higher plants. European Mol Biol Organisation J 6: 3901-3907.

17. Ditt RF, Kerr KF, Figueiredo PJ, Delrow L \& Nester EW (2006). The Arabidopsis thaliana transcriptome in response to Agrobacterium tumefaciens. Mol Plant Microbe, Interaction 19: 665-681.

18. Pruss GJ, Nester EW \& Vance V (2008) Infiltration with Agrobacterium tumefaciens induces host defense and development dependent responses in the infiltrated zone. Mol Plant-Microbe Interact 21(12): 1528-1538.

19. Veena JH, Doerge RW \& Gelvin SB (2003). Transfer of T-DNA and Vir proteins to plant cells by Agrobacterium tumefaciens induces expression of host genes involved in mediating transformation and suppresses host defense gene expression. Plant $J$ 35: 219-236.

20. Grosse-Holz F, Steven K, Svenja B, Farnusch K, Markus K \& Renier ALH (2018). The transcriptome, extracellular proteome and active secretome of agroinfiltrated Nicotiana benthamiana. Plant Biotech J 16: 1068-1084.

21. McIntosh KB, Hulm JL, Youn LW \& Bonham-Smith PC (2004). A Rapid Agrobacterium-mediated Arabidopsis thaliana transient assay system. Plant Mol Biol Reporter 22: 53-61.

22. Lawrence GJ, Finnegan EJ, Ayliffe M A \& Ellis JG (2005). The L6 gene for flax rust resistance gene is related to the Arabidopsis bacterial resistance gene RPS2 and tobacco viral resistance gene. Nature Plant Cell 7: 1195-1206.

23. McCullen CA \& Binns AN (2006). Agrobacterium tumefaciens and plant cell interactions and required for inter kingdom macromolecular transfer. Ann Rev Cel Div Biol 22: 101-27.

24. Grosse-Holz F, Steven K, Svenja B, Farnusch K, Markus K \& Renier ALH (2018). The transcriptome, extracellular proteome and active secretome of agroinfiltrated Nicotiana benthamiana. Plant Biotechnol J 16: 1068-1084.

25. Norkunas K, Robert H, James D \& Benjamin D (2018). Improving agroinfltration-based transient gene expression in Nicotiana benthamiana. Plant Methods 14: 71. 